\title{
mHealth and Health Analytics Interventions to support Patients with Advanced Chronic Kidney Disease
}

\author{
Ramsay Meiklem \\ Department of Computer and Information Sciences, \\ University of Strathclyde, \\ Glasgow, UK \\ ramsay.meiklem@strath.ac.uk
}

\begin{abstract}
Chronic kidney disease is a substantial global health burden, very significant independent risk factor for further morbidities, greatly decreases quality of life and has a very high associated cost to health systems. Patients experience complex treatment trajectories, high levels of treatment burden and can become frustrated and dissatisfied with their experience. I seek to investigate the potential of mobile health technologies to support advanced chronic kidney disease patients in their treatment, by iteratively developing and evaluating produced solutions with patients and experts, while working with NHS partners to form an implementation and deployment strategy for such solutions into routine practice.
\end{abstract} centred

advanced chronic kidney disease; patient portal; treatment burden; mobile health (mHealth); patient-

\section{INTRODUCTION}

I am a second year PhD candidate researching how mobile health (mHealth) and health analytics interventions can support patients with advanced chronic kidney disease (ACKD). Recent studies have shown treatment places a great burden upon patients physically and emotionally (Jones et al., 2017), with recommendations to facilitate safer and more effective, patient-centred care (Oliver et al., 2017). From these recommendations, I have worked to produce a prototype patient portal (Bouamrane et al., 2019), intended for use by haemodialysis patients, prior to my $\mathrm{PhD}$ and have continually developed and evaluated it with clinical and human-computer interaction $(\mathrm{HCl})$ experts.

Now I seek to evaluate and refine the current iteration with patients as part of my research, while reviewing the literature to gather insight and lessons from other technology-based interventions for similar user groups. Building on previous work, I have begun investigating how health analytics of existing patient treatment data and mHealth solutions can be used to reduce the complexity of treatment pathways and support patients when understanding their condition and treatment options.

\section{RESEARCH TOPIC}

Chronic kidney disease is a substantial global health burden, recognised as a very significant independent risk factor for further morbidities, substantial decreases in quality of life and having a very high associated cost to health systems (Hill et al., 2016; Fukahara et al., 2003; Cleary and Drennan, 2005). Treatment options and trajectories for patients with chronic kidney disease are complex and subject to important and possibly unjustified variations.

Haemodialysis is one such treatment option, performed when the kidneys no longer function normally, such as in the case of chronic kidney disease (Stenvinkel, 2010). There are various treatment methods currently used in clinical practice, which make for complex patient pathways and complications associated with modalities are also common (Aitken et al., 2017; Feldman et al., 1996). Haemodialysis treatment has substantial emotional impact on patients themselves as well as their families and social relationships (Jones et al., 2017) and patients are often frustrated and dissatisfied with their personal experiences of treatment (Oliver et al., 2017). These findings highlight the high treatment burden (the work placed upon the patient as result of their healthcare and the impact upon their wellbeing (Eton et al., 
2012)), where the term work refers to both treatment and self-care for a condition(s) (Gallacher et al., 2018)) experienced by haemodialysis patients and the complexity of treatment is reflected in the information work (defined as "quest for, receiving of, and the passing of information") of patients, investigated by Burgess et al. (2019), where they noted dynamic shifts in the information work as patients as they progressed in their healthcare and understanding of their condition.

While there is no immediate solution for high treatment burden, there are proposed measures to reduce such burdens. May et al (2009) proposed the term "minimally disruptive medicine" referring to forms of effective healthcare and provision of service purposed to minimise the treatment burden upon the patient while furthering their healthcare goals. Various health condition specific measures of treatment burden have been produced from studies, including a measure for end-stage renal disease (ESRD), the stage of chronic kidney disease where the kidney function is so reduced dialysis treatment or transplantation is required (Murphy et al., 1985).

With the growth and adoption of digital health and mHealth, such technology-based interventions could support haemodialysis patients with their treatment and reduce the complexity of treatment pathways and work required to understand them.

\section{RESEARCH AND WORK}

\subsection{Prior to current research}

I sought to gather and synthesise the opinion of clinical and Human Computer Interaction ( $\mathrm{HCl})$ domain experts to establish a set of initial design requirements in order to test the feasibility of developing a digital aid to support haemodialysis patients in the course of their treatment, an electronic haemodialysis patient portal (Bouamrane et al., 2019). We concluded that while some of the synthesised design requirements may be applicable to all patient portals or similar technology-based interventions, idiosyncratic requirements inherent to the condition is essential for successful deployment of a patient portal designed for targeted patient groups. This work was completed prior to the beginning of my PhD, initially as a dissertation submitted in part fulfilment of the requirements for a postgraduate degree in digital health systems. In this project I was responsible for the design and running of interviews and focus groups with clinical and $\mathrm{HCl}$ experts for requirements gathering and evaluation purposes, synthesising design requirements and themes from transcribed discussions and the development of an
Android native mobile application to host the prototype patient portal and required functionalities.

\subsection{Work completed to date}

Work to further evaluate and refine the current iteration of the portal has continued with clinical experts, specifically NHS partners with whom I am forming deployment and implementation strategies for integrating the patient portal into routine practice and into ongoing studies. For example, the patient portal is being utilised within a validation study to validate a vascular access quality of life measure, where the portal is hosting the new tool and the two validated measures and recording the response data automatically.

A usability study with current and former dialysis patients was ongoing prior to restrictions as result of the COVID-19 pandemic and advice from our NHS partners, as this population are very high risk and would likely experience enough stress and worry while having to attend regular treatment sessions while in a pandemic. However, I hope to resume this evaluation when appropriate, potentially in an accessible online format.

As I have become more familiar in my research topic and developed a technology-based intervention to recue treatment burden of ACKD patients, I started to ask if there were similar technologies for other health conditions with similar levels of treatment burden and how effective they were. In order to answer this question, I completed a protocol for a scoping literature review in order to answer my research question:

'What patient-centred, technology-based interventions have been implemented to support patients with high treatment burden?'

\subsection{Ongoing and future work}

As discussed in the previous section, I plan to conduct a scoping review. With a clear protocol established, I aim to identify and gather insights into how to design, develop, evaluate and implement effective, patient-centred and technology-based interventions to support patients with high treatment burden, relevant and useful to both the $\mathrm{HCl}$ and clinical communities.

There is potential for evaluation of the current patient portal mobile application to restart with patients and experts, in an online or remote format which does not require participants to physically attend a session. I am currently formally testing the current version of the application and will seek to complete usability and heuristic evaluations if appropriate. I have begun work with clinical and $\mathrm{HCl}$ experts to gather design requirements and considerations for designing data visualisations to convey patient pathway information. While 
originally an initial design requirement synthesised from the patient portal feasibility study, the need for an interactive guide to patient pathways and clarity of treatment options is clear in the literature. Transcription of audio from an online design workshop with experts has been completed and will be followed by coding of the transcription and analysis of draft visualisations produced by participants of the workshop, from an existing patient treatment journey dataset.

\section{CONCLUSION}

Chronic kidney disease patients must endure both their condition and the burden of their treatment they require to prolong their health. mHealth and health analytics have the potential to support patients in their treatment. I have established such solutions are feasible in previous work and now seek to validate such interventions with patients themselves and identify key examples of similar technologies for other conditions. From this research, I hope to contribute meaningful information to both the clinical and $\mathrm{HCl}$ communities on how we can develop, evaluate and deploy effective and patient-centred mHealth interventions for those living with chronic conditions and high treatment burden.

\section{REFERENCES}

Aitken, E., Thomson, P., Bainbridge, L., Kasthuri, R., Mohr, B. and Kingsmore, D., 2017. A randomized controlled trial and costeffectiveness analysis of early cannulation arteriovenous grafts versus tunneled central venous catheters in patients requiring urgent vascular access for hemodialysis. Journal of Vascular Surgery, 65(3), pp.766-774.

Bouamrane, M.M., Meiklem, R., Dunlop, M., Kingsmore, D., Thomson, P., Stevenson, K. and Greenwood, S., 2019, June. Haemodialysis electronic patient portal: a design requirements analysis and feasibility study with domain experts. In 2019 IEEE 32nd International Symposium on Computer-Based Medical Systems (CBMS) (pp. 212-216). IEEE.

Burgess, E.R., Reddy, M.C., Davenport, A., Laboi, P. and Blandford, A., 2019, May. " Tricky to get your head around" Information Work of People Managing Chronic Kidney Disease in the UK. In Proceedings of the $2019 \mathrm{CHI}$ Conference on Human Factors in Computing Systems (pp. 1-17).

Cleary, J. and Drennan, J., 2005. Quality of life of patients on haemodialysis for end-stage renal disease. Journal of advanced nursing, 51(6), pp.577-586.
Eton, D.T., de Oliveira, D.R., Egginton, J.S., Ridgeway, J.L., Odell, L., May, C.R. and Montori, V.M., 2012. Building a measurement framework of burden of treatment in complex patients with chronic conditions: a qualitative study. Patient related outcome measures, 3, p.39.

Feldman, H.I., Kobrin, S. and Wasserstein, A., 1996. Hemodialysis vascular access morbidity. Journal of the American Society of Nephrology, 7(4), pp.523-535.Eton, David T., et al. "Building a measurement framework of burden of treatment in complex patients with chronic conditions: a qualitative study." Patient related outcome measures 3 (2012): 39.

Fukuhara, S., Lopes, A.A., Bragg-Gresham, J.L., Kurokawa, K., Mapes, D.L., Akizawa, T., Bommer, J., Canaud, B.J., Port, F.K. and Held, P.J., 2003. Health-related quality of life among dialysis patients on three continents: the Dialysis Outcomes and Practice Patterns Study. Kidney international, 64(5), pp.1903-1910.

Gallacher, K.I., May, C.R., Langhorne, P. and Mair, F.S., 2018. A conceptual model of treatment burden and patient capacity in stroke. BMC family practice, 19(1), p.9.

Hill, N.R., Fatoba, S.T., Oke, J.L., Hirst, J.A., O'Callaghan, C.A., Lasserson, D.S. and Hobbs, F.R., 2016. Global prevalence of chronic kidney disease-a systematic review and metaanalysis. PloS one, 11(7), p.e0158765.

Jones, D.J., Harvey, K., Harris, J.P., Butler, L.T. and Vaux, E.C. (2017). Understanding the impact of haemodialysis on UK National Health Service patients' well-being: A qualitative investigation. Journal of Clinical Nursing, 27(12), pp.193-204.Oliver, Scott W., et al. "A national appraisal of haemodialysis vascular access provision in Scotland." The journal of vascular access 18.2 (2017): 126-131.

May, Carl, Victor M. Montori, and Frances S. Mair. "We need minimally disruptive medicine." Bmj 339 (2009): b2803.

Murphy, S.P., Powers, M.J. and Jalowiec, A., 1985. Psychometric evaluation of the hemodialysis stressor scale. Nursing Research.

Oliver, S.W., Campbell, J., Kingsmore, D.B., Kasthuri, R., Metcalfe, W., Traynor, J.P., Fischbacher-Smith, D., Jardine, A.G. and Thomson, P.C. (2017). A national appraisal of haemodialysis vascular access provision in Scotland. The Journal of Vascular Access, 18(2), pp.126-131.

Stenvinkel, P., 2010. Chronic kidney disease: a public health priority and harbinger of premature cardiovascular disease. Journal of internal medicine, 268(5), pp.456-467. 